\section{From Lab to Field Extending the Architectural Design Studio to Integrate Emerging Technologies}

Corneel Cannaerts

KU Leuven Faculty of Architecture

\section{EXTENDING THE STUDIO}

The architectural design studio, as a place for educating future practitioners, is faced with two necessary dissociations: the distance from practice and its futurity. While the responses in architectural education have been varied ${ }^{1}$, the question of how to integrate emerging technologies seems to further sharpen these dissociations. This paper discusses the MMlab and Fieldstation studio, two learning environments set up as extensions of the design studio aiming to question the impact of emerging technologies on architecture. These extensions are particular ways of responding to the dissociations between the design studio and practice and its futurity: through hands-on experimentation with emerging technologies and questioning their relevance for architectural practice and culture, and by exploring the impact of technologies on the environments in which we operate as architects, deliberately looking for places and sites where emerging technologies manifest themselves with a particular urgency. The argument builds on a number of design studios, workshops and elective courses, it discusses two case studies in detail and describes the shift from lab to field in terms of subject matter, spatial setting and pedagogical approach.

\section{SETTING UP THE LAB}

The MMLab² was established at the Faculty of Architecture of KU Leuven in 2010 as a place for hands-on experimentation with digital fabrication and computation as drivers for architectural design. The lab was set up based on the experience in using computational design and digital fabrication as design media, i.e. exploring how these emerging technologies mediate design processes. Over a period of nine years the MMlab has grown, relocating to the former church of the Sint-Lucas campus in Ghent. The MMlab provides lasercutting, cnc milling, 3D printing, prototyping and woodworking facilities. The teaching and research practice established at the lab developed from technology-based tutorials to design-driven elective courses and workshops, shifting from the acquiring of technical skills to questioning the impact of technologies on the practice and culture of architecture.

Through the research and experimentation at the MMlab a particular approach to computational design and digital fabrication was established: Rather than looking at digital fabrication for closure, i.e. closing the gap between the designed and fabricated artifacts, it explores how the encoding of design in a file, the operations of the machines and the materials they work with, contribute to the fabricated artifact. In other words, computation and digital fabrication were approached as design media, uncovering their agency in design and fabrication processes, leading to novel design outcomes.

The argumentation for this was developed into the doctoral thesis Negotiating Agency: Computation and Digital Fabrication as Design Media $^{3}$, the approach was further developed as a guest researcher at the Aarhus School of Architecture ${ }^{4}$. In parallel, this was explored through collaborations in architectural practice and built architectural projects ${ }^{5}$. The MMlab has hosted several workshops, elective courses and research projects, below we will discuss one specific workshop in detail as it clearly demonstrates the developed approach. 


\section{UNRULY SURFACES}

Unruly Surfaces was an intensive four-day workshop organized as a masterclass ${ }^{6}$ and symposium at the MMlab in 2018, within the framework of the Hello Robot exhibition at the Design Museum Ghent? The workshop introduced participants in the process of robotic fabrication from design ideation to material artifact. After an introduction into parametric modeling and programming of a collaborative robotic arm, the participants experimented with the fabrication process, next to using other design media such as sketching and modeling. In addition to the geometric representation of the design model, the fabrication process requires supplementary information, e.g. fabrication process parameters and machine and material specifications. The materiality of the fabricated artifact is thus dependant on the encoding of this information and on the machine and material properties. Through the iterative process of experimentation through fabrication, the design is refined to take into account material properties and qualities, fabrication constraints, means of connecting and assembling (See Figure 1).

The workshop was based on robotic hotwire cutting ${ }^{8}$, a process that uses a heated nichrome wire to cut through extruded polystyrene foam. The title of the workshop is a play on ruled surfaces? geometries that can be described through a sequence of straight lines, similar to the hot wire in robotic hotwire cutting. Notwithstanding this analogy between the geometric representation and fabrication process, the workshop explicitly explored the discrepancies between the idealized geometry and the unruly material outcomes. These discrepancies result from material properties, even within an industrially produced material such as EPS foam, density and humidity can vary substantially. Fabrication processes parameters affect the resulting materiality, in particular, the speed of cutting: When moving faster there will be tension on the hotwire, resulting in deformation and the wire no longer being a straight line. On the contrary, when moving slower or even stopping the wire will melt away more material than described by the ruled surface it follows.

Participants designed and constructed three large scale installations whose materiality results from experimenting with the specific fabrication process, allowing the agency of encoded information, machine and material to contribute to the design outcome. Spiral screen (see Figure 2) used the maximum angles of the sixth joint of the robotic arm to cutting spirals while moving up and down a block of foam. Stereotomic wall (see Figure 1-2), referencing stereotomy a technique for cutting stone, explored cutting volumetric blocks assembled so that only two blocks meet in each joint. Inverse totem (see Figure 3-4) explored the difference between the smooth ruled surfaces and more erratic fluted surfaces in an installation with a smooth inside and a richly textured outside.

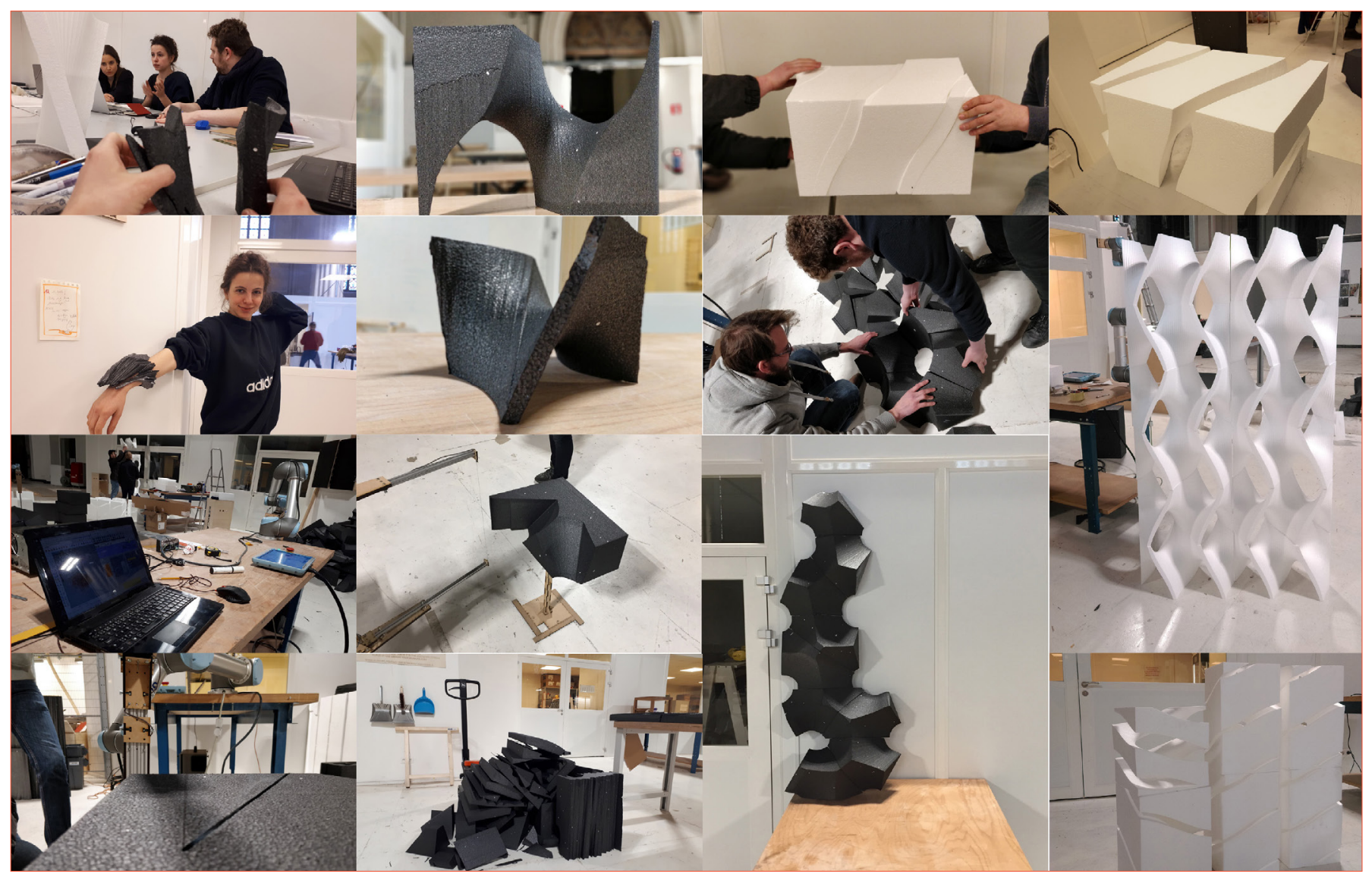

Figure 1. Uruly Surfaces, experimentation. 
The use of ruled surfaces in architectural design allows for an expressive formal language that is straightforward to construct as it can be made from straight elements. Robotic hotwire cutting is a well-established digital fabrication technique with several examples in architecture both in research and practice ${ }^{10}$. The technique is mainly used to fabricate formwork for casting concrete and heralded for its speed compared to other subtractive fabrication processes such as CNC milling ${ }^{11}$. In addition to the parameters of constructability and speed, the Unruly Surfaces workshop explored material expressions deriving from the agencies of materials and machines that exceed the geometric description.

This approach to digital fabrication questions the impact of emerging technologies on the role of the architect, the involvement in processes of making, the use of design media and authorship. This process can be described as a negotiation with the agencies at play, rather than looking for control the encoding of information, the fabrication technique and material properties all contribute to the materiality of the artifacts. The resulting installations are obviously prototypical, i.e. the workshop focused on a subset of design questions and did not engage the full complexity of an architectural project. This temporary isolation from the world, the deliberate freedom to focus on material articulation, is what the lab provides, a place to develop an architectural language and expression inherent in the fabrication technology through hands-on experimentation.
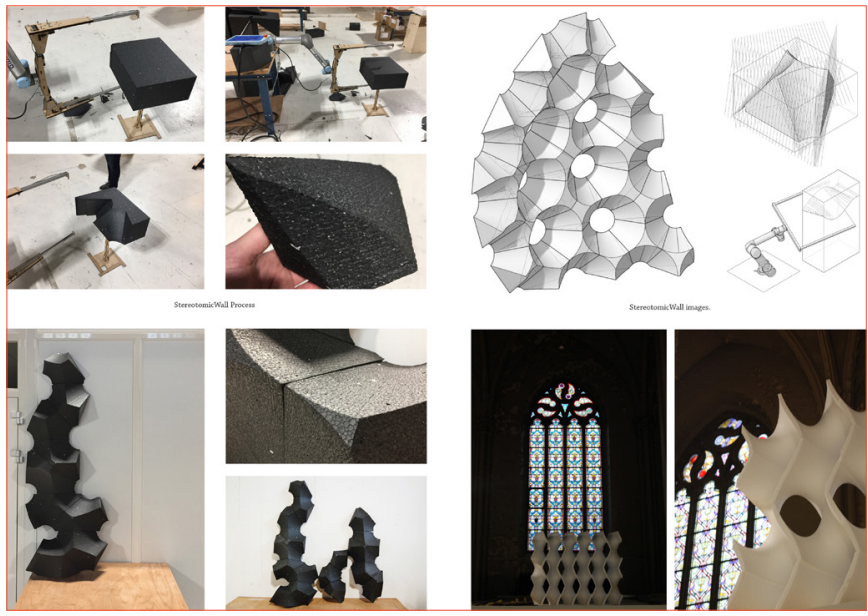

Figure 2. Uruly Surfaces, design itteration

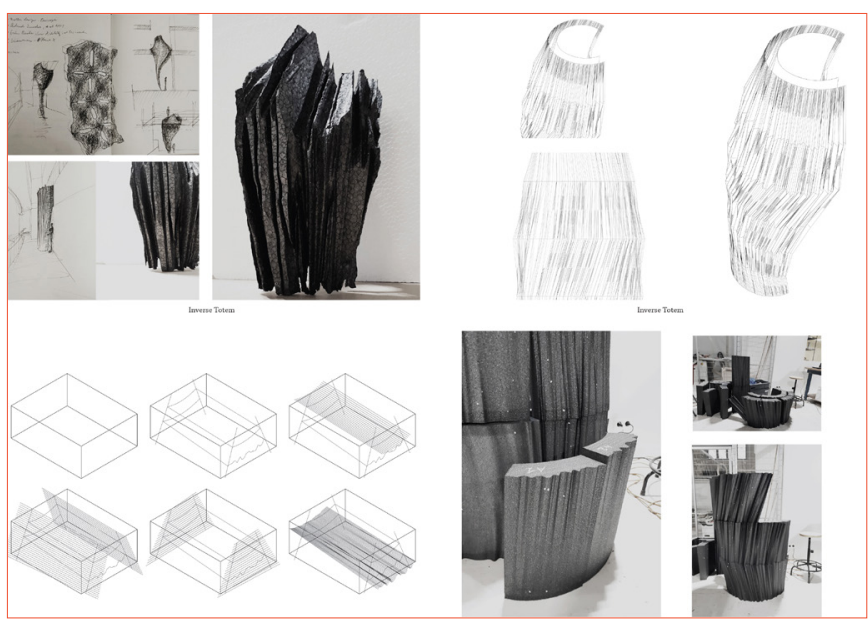

Figure 3. Uruly Surfaces, fabrication proces.

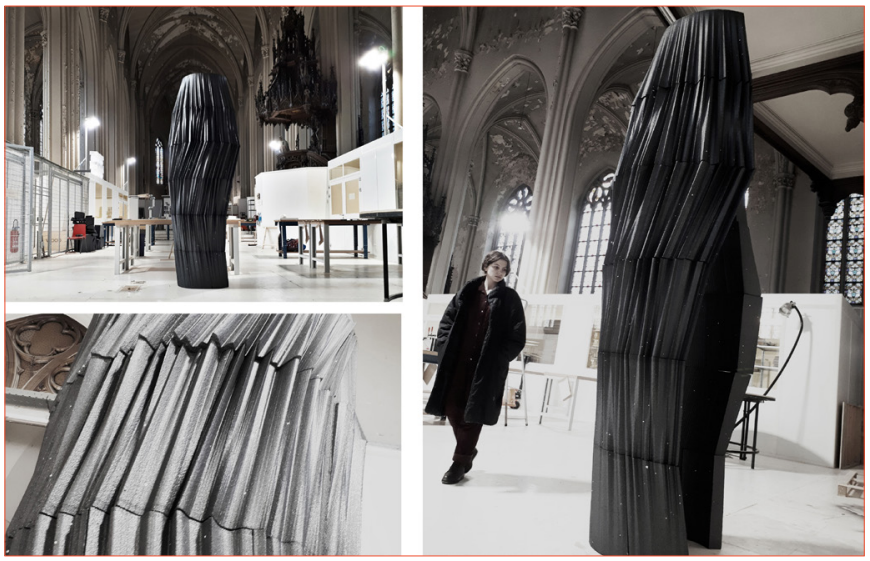

Figure 4. Uruly Surfaces, resulting installation. 


\section{INTO THE FIELD}

Architects, both in practice and in academia have approached digital technologies mainly as an extension of their toolbox, developing digital means for drawing, modeling, calculating and communicating architectural ideas. The questions addressed at the MMlab while shifting from technology-based teaching towards exploring the impact of digital technologies on architectural practice and culture were still looking through the lens of technology as a medium, i.e. technology as a means of designing and fabricating architectural artifacts. While establishing the teaching and research agenda of the lab, it became gradually more clear that emerging technologies are increasingly provoking new challenges and questions to architectural practice and research, not through their agency as design media but in the impact they have on the environment at large.

The environment in which we operate as architects are increasingly saturated with digital technologies: internet-of-things, global communication and transportation technologies, mobile devices, increased satellite coverage, location-based services, ubiquitous computing... ${ }^{12}$ What distinguishes this technological layer or technosphere ${ }^{13}$ from previous human-made infrastructures is the interconnectedness of devices, people and environments. This 'accidental megastructure' is not designed but emerges as a stack ${ }^{14}$ of interrelated fields and gives rise to radically new geographies. ${ }^{15}$ These connected technologies heralded for their potential to enhance our build environments, improve our lives and democratize access to information, come with a dark side; its interfaces are enabled through resource depletion, cheap labor, exclusion and pollution ${ }^{16}$. The material impact of our collective technologies is so extensive it will leave a lasting imprint on our planet, prompting geologist to established the Anthropocene, as a new geological epoch. ${ }^{17}$

\section{FIELDSTATION STUDIO}

Fieldsation studio was set-up by Michiel Helbig and Corneel Cannaerts in response to the challenges to architectural practice and culture posed by the technosphere and the Anthropocene. Fieldstation studio was set up as a master studio at the Faculty of Architecture of the $\mathrm{KU}$ Leuven in 2016. It is part of an academic

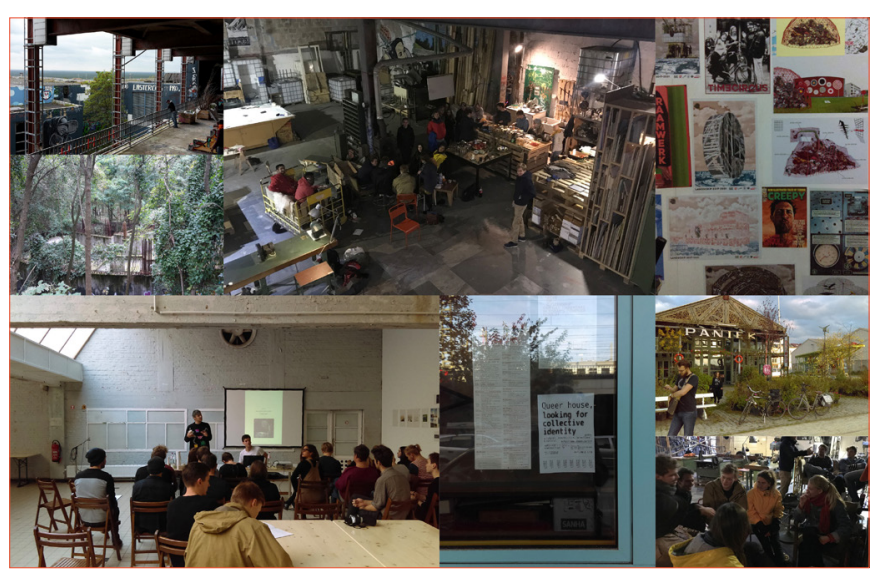

Figure 5. Fieldstation Studio, fieldtrip design office, a type of learning environment confronting research and education with real-world challenges through collaboration with external partners. Fieldstation studio is a node in the international Fieldstations network of architects, artists, scientist and activists exploring new models for architecture in relation to the Anthropocene and the technosphere. ${ }^{18}$ The network consists of a growing number of nodes in Berlin, Basel, Ghent, Vielitz and Sicily, it organizes workshops, exhibitions and summer schools ${ }^{19}$ and lectures, where members discuss projects and share knowledge and engage in public debates.

The network initially had its headquarters at Teufelsberg ${ }^{20}$, an artificial hill constructed from the rubble of Berlin after the second world war, on the site of the Nazi military-technical school designed by Albert Speer, operating as the base of the NSA spy station from 1961 till 1992. As the buildings on Teufelsberg and their layered history demonstrate, architecture cannot be reduced to the constellation of material elements and is subject to environmental, technological and cultural changes ${ }^{21}$. The built reality is only one layer that makes up the environments we inhabit, it is embedded within other material and immaterial layers, and it contributes to larger economic, material, environmental, informational and infrastructural systems. Within the design studio, this expanded field, this constantly changing, layered and hybrid environment is seen as the context architecture operates in and actively engages with.

Fieldstation Studio aims to engage with the complex reality described above by rethinking our modes of operation and our position as architects designing embedded in this layered and hybrid environment. The studio investigates the potential of architecture as a medium to explore disrupt and raise questions rather than solving them. The studio proclaims that architects should proactively engage the complex reality of today rather than passively waiting for design briefs and projects. The design studio trains students in taking a position within contemporary fields and provides them with a platform for developing their future practice. Additional elective courses provide students with the necessary critical tools, skills and design media. The tools of choice are design fiction, spatial narratives, speculative media, imagineering, hacking and critical making. The studio operates as a collective practice, students are encouraged to actively participate in

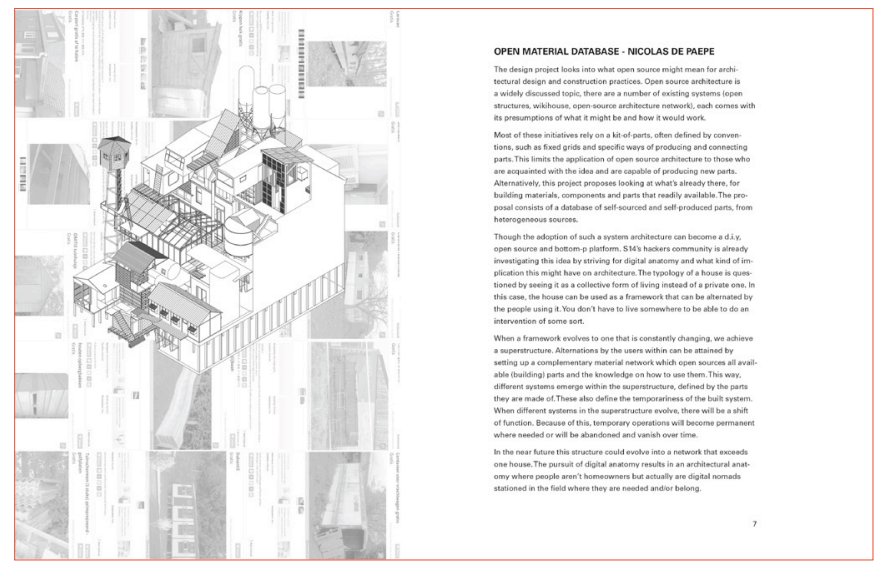

Figure 6. Hacking: Open Material Database (Nicolas De Paepe). 
the organization and content of the studio, breaking out of the confines of academic architectural education. The studio undertakes fieldwork and actively seeks encounters with practitioners, thinkers, makers, hackers, architects and artists operating in similar fields, to exchange alternative practices, to share experiences and ideas.

The studio runs for a period of fourteen weeks, starting with a field trip, an intensive workshop week around midterm, and a public exhibition and debate at the finals. The brief is split into two parts: Starting from lectures, study trips, reading, presenting and discussing examples and theoretical texts, the aim of the first part is to collectively identify, map and categorize the potential of a particular theme or urgency for architecture. In groups of three, students develop a field guide, focussing on a particular topic within the larger theme of the studio and explore this topic through collecting references, and designing prototypes that demonstrate its relation and relevance for architecture. In the second part, an extensive workshop introduces students to a concrete situation and site where the theme of the studio manifests itself with a certain urgency. The concepts and prototypes resulting from the first part are further developed and implemented through the confrontation with this concrete situation and the questions and potential it raises. The studio is organized in the first semester of the four-semester master program and aims to open the scope of what future architectural practice might become, as such students are free to interpret the brief in terms of scope, scale, program and strategy as a first step in developing their personal position and practice.

The studio has run for three editions each focussing on a different theme within the larger interest in architecture's position within the complex reality of today. The title of the studios so far have been Hacking the Expanded Field of Architecture (2016), Shifting Borders (2017) and Architecture and Platforms (2018), and we are planning a studio on Architecture and Automation (2019) and Precarity and Luxury (2020). Through these, we are unpacking various aspects of how technology is increasingly impacting the environments in which we operate as architects. Rather than describing all of the studios I will discuss one in detail and outline the understanding that has been developed through the different studios in general.

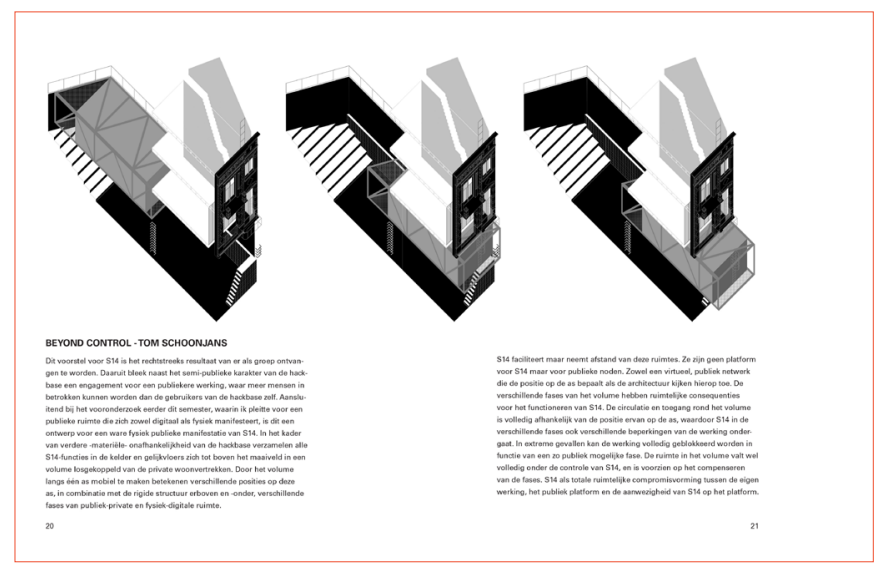

Figure 7. Hacking: Beyond Control (Tom Schoonjans)

\section{HACKING THE EXPANDED FIELD OF ARCHITECTURE}

The 2016 edition of fieldstation studio looked into hacking as an approach to architecture and its potential to expand the field of spatial practices. Hacking was understood in the broadest sense of understanding a specific technology or system, revealing its affordances and using that knowledge to make it operate beyond its intended use. This approach was applied to different aspects of architectural practice and culture, from processes of design and building to the operation and functioning of buildings to material articulation. We collaborated with S1422, a hackbase in Antwerp, a place that provides co-living in addition to operating as a hackerspace organizing workshops and events. S14 is based in a regular row house and comprises, next to the hackbase, of a collective allotment garden and a boat in the harbor of Antwerp. Aspects of its architecture are organized within a hackers ethos, as can be read in the manual for the house. Different user roles, inhabitant, lodgers, and passer-by, come with degrees of access to collective and private spaces, and different responsibilities. Through small interventions the house has been made more open: a platform in the ground floor room facing the street acts as a stage, the window on the first floor becomes a vitrine, a secondary staircase and climbing stones on the façade open up different routes for circulation. The interventions are done in a d.i.y. fashion often as part of a workshop, using materials in unconventional ways.

Field guides focussing on a specific interest of hacking in relation to architectural design were developed by groups of students in the first phase of the studio. To provide some examples: one group of students developed a field guide into open-source architecture ${ }^{23}$, based on references like the open structures project, open-source architecture, global village construction set, open-source ecology, wikihouse ${ }^{24} \ldots$ they developed a kit of parts that could be combined into various temporal and mobile structures. Another group of students was interested in how buildings communicate and engage a larger public, collecting examples of propaganda architecture, advertisement, media facades, and event architecture, they looked into how this communication could be hacked and appropriated.

Students individually developed an architectural proposal for S14 in the second phase of the studio, further exploring and applying the ideas developed in the field guide.

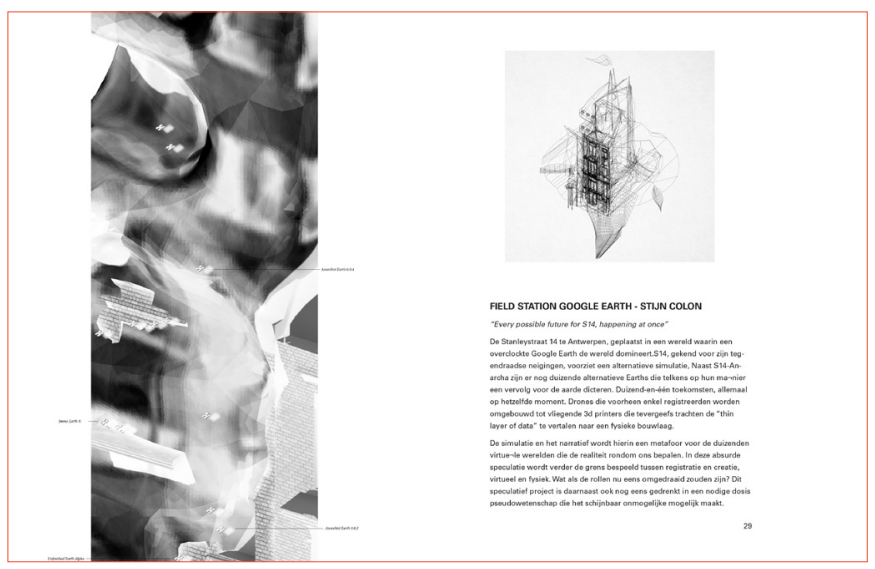

Figure 4. Hacking: Google Earth (Stijn Colon) 
Nicolas De Paepe developed Open Material Database, a website that collects freely available building materials from online platforms, describing their dimensions, materials, state... Adopting an opensource strategy to deal with a heterogeneous set of material artifacts and architectural parts. As a case study, he designed an extension to the house with the materials he collected for one month, into an aggregate superstructure, further extrapolating the d.i.y. building practices of S14.

Tom Schoonjans' project Beyond Control was inspired by the different kinds of access to S14, and the relation of the building with its direct surroundings. He designed a moving structure on the ground floor that depending on its position would change the accessibility of the house, revealing a public basement or locking down some parts of the building. The adaptable accessibility is also taken up in the interior where moveable furniture organizes different uses of the space.

Stijn Colon's project titled Fieldstation Google Earth explored the relationship between the earth and google earth as its digital counterpart. While at the moment google earth is updated every few years, lagging behind physical earth. As a response to the increased refresh rate, Stijn speculated on what might happen if google earth would catch up or even evolve faster than physical earth, running simulations of various versions of the earth simultaneously, he designed a version of the house where these conflicting future scenarios would battle of which one would be materialized.

The studio wrapped up with a discussion at S14 and an exhibition where both field guides and final projects where presented. The radical openness of S14 as a community - students got free access to the house - e.g. the alternative practices of constructing and using the house, the collective allotment garden and boat, inspired students to come up with architectural proposals often highly personal and original. The confrontation with spatial practices and strategies on the fringe or just outside of contemporary architectural practice, allowed students to speculate on their own postion and practice. Overall the field guides and individual proposals demonstrate that hacking is a viable architectural strategy, that can inspire novel design outcomes that expand the field of operations for architectural practice.

\section{SHIFTING TECHNOLOGICAL AGENCY}

The two extensions of the design studio discussed, the lab and the field, emerged from being situated both within practice and academia. The starting point for these extensions is the experience of the impact of technologies on architectural practice, leading to the assumption, or hunch, that the role of emerging technologies is not as clear as it is promoted to architectural practitioners and students, i.e. that technologies are not neutral means for designing and fabricating architecture, but that technologies bring their own agencies. This assumption was substantiated firstly through setting up the lab, uncovering the agency of technologies as design media during design processes, and secondly by venturing into the field, beginning to unpack the agency of emerging technologies in the environment at large.

The lab as a place for experiment and research within architecture is predicated on temporarily isolating and focussing on certain aspects of architectural design while making abstraction of others.
This dissociation, the freedom of not having to incorporate all of architectural designs parameters, works liberating and allows for iterating on a subset of design parameters with precision and speed. Digital fabrication, in particular, is of interest here as it short-circuits representation, and allows for materiality and making to inform design processes, rather than being an afterthought. A substantial amount of the design production in digital fabrication labs remains prototypical, i.e. the developed procedures, fabricated artifacts and discovered material qualities hold the promise to be applicable in architectural practice, if not today, then in the near future.

Encountering real-world challenges through field trips, engaging with alternative spatial practices and strategies, exploring places where contemporary phenomena manifest themselves with urgency, as a pedagogical appraoch aims to bridge the dissociation with future architectural practice by bringing in themes and topics on the fringe of or outside of the field of contemporary architectural practice. While these themes might seem overwhelmingly complex and large for students, field station studio provides them a way to incorporate them within their architectural practice, by focussing on specific subtopics and providing concrete situations and sites where these themes manifest themselves. In that sense the design production of field station studio is likewise prototypical, i.e. students produce architectural proposals and sketch designs, that don't necessarily take into account the whole complexity of the engaged themes and sites, but highlight the architectural potential of emerging technologies and phenomena.

The shift from lab to field can be described in terms of the role technology plays within the architectural design studio, shifting from technology as a medium or tool, to technology as subject matter or driver for contemporary phenomena. However, both question the agency of emerging technologies and their impact on future practice, as such they are different ways of bridging the dissociations between practice, its futurity and the architectural design studio.

\section{ACKNOWLEDGEMENTS}

The work described in this paper was made possible by Michiel Helbig, Christophe Barlieb, Marthe Van Dessel, people from S14, participants of the Unruly Surfaces workshop (Adam Novotnik, Anja Smits, Anka Eckels, levgeniia Koval, Katja Lorencic, Kevin Saey, Remco Vanhooren and Sven Desmet), participants of Hacking the Expanded Field of Architecture (Aaron Derie, Eliza De Meyer, Georgieva Ramona, Kevin Saey, Lieselot Lust, Maarten De Smet, Marie Malingreau, Marine Boey, Nicolas De Paepe, Tom Schoonjans, Tsvetan Karamochev, Shervin Sheikh Rezaei, Stijn Colon, Vincent Nulens and Younes Ben Amar). 


\section{Notes}

1. See amongst others Spiller, Neil, and Nic Clear, editors. Educating Architects: How Tomorrow's Practitioners Will Learn Today. Thames \& Hudson, 2014.

2. http://llumen.be/MMlabKUL/, accessed June 24, 2019.

3. Cannaerts Corneel, Burry Mark. (sup.), Verbeke Johan. (cosup.) "Negotiating Agency: Computation and Digital Fabrication as Design Media" (PhD diss., RMIT University, 2015).

4. Cannaerts Corneel. "Substantiating Lines: Threads, Folds \& Traces". In: CA2RE: Conference For Artistic and Architectural (Doctoral) Research Proceedings. (294-305). Presented at the CA2RE: Conference For Artistic and Architectural (Doctoral) Research, Aarhus School of Architecture, 2018.

5. Cannaerts Corneel, Hoffmann Holger. "Grounding Associative Geometry: From Universal Style Towards Specific Form". In Research Culture in Architecture. Cross-Disciplinary Collaboration. Editors: Loeopold, Cornelie, Robeller, Christopher, Weber, Ulrike. Birkhauser 2019.

6. http://rxd.architectuur.kuleuven.be/projects/robotic-fabricationmasterclass-and-symposium/, accessed on June 24, 2019.

7. https://www.designmuseumgent.be/agenda/hello-robot, accessed on June 24, 2019

8. A technique developed in architecture at Taubman College of Architecture \& Urbanism (See Matter \& Making in Glynn, Ruairi, and Bob Sheil (eds). Fabricate: Making Digital Architecture. Riverside Architectural Press / ABC Art Books Canada, 2011.) TU Delft (Verde, Marco, Hosale, Markdavid and Feringa, Jelle. "Investigations in Design \& Fabrication at Hyperbody" In: Glynn, Ruairi, and Bob Sheil. Fabricate: Making Digital Architecture. Riverside Architectural Press / ABC Art Books Canada, 2011.) and Aarhus School of Architecture (Sondergard, Asbjorn and Feringa, Jelle. "Fabricating Architectural Volume: Stereotomic Investigations in Robotic Craft" In: Gramazio, Fabio, Matthias Kohler, and Silke Langenberg. Fabricate Negotiating Design and Making. E TH Honggerberg Zurich, 2014.

9. There are several applications of ruled surfaces in architecture, most significantly Antoni Gaudi in his later work as extensively discussed by Mark Burry. See Burry, Mark. "Geometry Working Beyond Effect." In Architectural Design 81, no. 4 Special Issue: Mathematics of Space (July 2011): 80 -89. https://doi. org/10.1002/ad.1272.

10. For applications in academia see refrences in footnote 8 , for applications in architectural practice see Asbjørn Søndergaard and Jelle Feringa. "Scaling Architectural Robotics: Construction Of The Kirk Kapital Headquarters" In: Fabricate, Achim Menges, Bob Sheil, Ruairi Glynn, Marilena Skavara, Universität Stuttgart, and Bartlett School of Architecture and Planning, eds. Fabricate: Rethinking Design and Construction. London: UCL Press, 2017.

11. For a comparison between cnc milling and robotic hotwire cutting, see McGee, Wes, Jelle Feringa, and Asbjørn Søndergaard. "Processes for an Architecture of Volume." In Rob | Arch 2012, edited by Sigrid Brell-Cokcan and Johannes Braumann, 62-71. Vienna: Springer Vienna, 2013. https://doi. org/10.1007/978-3-7091-1465-0_5

12. Oosterman, Arjen, Lilet Breddels, Leaonardo Dellanoce (eds). Volume 51 - Augmented Technology. Archis, 2017.

13. The term was coined by Peter Half in 2014 (see Haff, Peter. K. Technology as a Geological Phenomenon: Implications for Human Well-Being. Geological Society, London, Special Publications, vol. 395, nr. 1, 2014, pp. 301-09), refrening to all humanity and man made structures, we use the term in a more narrow sense refrenign to the recent emergence of digital technology as a global spatial agent.
14. Bratton, Benjamin. The stack: on software and sovereignty. MIT Press, 2015.

15. Mattern, Shanon, Cloud and Field, On the resurgence of "field guides" in a networked age. Places journal, August 2016. https:// doi.org/10.22269/160802

16. Young, Liam \& Unknown Fields Division, eds. Tales from the Dark Side of the City, AA Publications 2016.

17. Turpin, Etienne, ed. Architecture in the Anthropocene: Encounters among Design, Deep Time, Science and Philosophy. Open Humanities Press, 2013.

18. For an overview of the network the agents involved and the different nodes see http://fieldstations.net/

19. Several workshops and summerschools were organised: Sense, Adapt, Creaate http://fieldstations.net/sense-adapt-create/, If This(), Then(Keephouse); http://fieldstations.net/wekeephouse/

20. Smith, Chris L., and Benjamin Jay Shand. "Architectural Wounds: Teufelsberg." In: Architecture and Culture 4, no. 2 (May 3, 2016): 185-92. https://doi.org/10.1080/20507828.2016.1189213.

21. Anderson, Benedict. Buried City, Unearthing Teufelsberg: Berlin and Its Geography of Forgetting. London and New York: Routledge, Taylor \& Francis Group, 2017.

22. This is a hackerbase organisedin Antwerp, see http://www.ooooo. be/S14/, the manual can found here: http://www.ooooo.be/ etherhtml/e2h.php? =S14

23. Ratti, Carlo, and Matthew Claudel. Open Source Architecture. London: Thames \& Hudson, 2015.

24. See: https://openstructures.net/,https://www. opensourceecology.org/gvcs/, https://www.wikihouse.cc/, all accessed on June24, 2019 